\title{
URBAN MOBILITY AND IOT: SMART SOLUTIONS FOR SUSTAINABILITY
}

\author{
Hannelore Nehring, Julhiano Glanert de Castro and Paulo Raimundi \\ Centro Universitário de Brusque - UNIFEBE, Brazil
}

\begin{abstract}
Internet of Things or IOT is the term used as a mean of connecting every device through the world wide web and by doing so, making use of the information present in the environment and that is not being consumed or processed in a computerized way yet, through this concept of new technologies it is possible to draw diverse essential alternatives to make our mobility in urban centers more intelligent and sustainable. The objective of this article is to make a definition of the terms, highlight the possibilities this technology presents us and suggest alternative solutions applicable to day-to-day situations that can positively and directly affect the direction of urban mobility in the near future, making it more computerized, technological and smart.
\end{abstract}

\section{KEYWORDS}

IOT, Mobility, Urban, Sustainability, Technology

\section{INTRODUCTION}

Nowadays our society is becoming denser, more populous and with more motor vehicles, and as a consequence the reality of urban mobility and quality of life became increasingly chaotic and insufficient to accommodate the society needs, so the capturing and processing of existing information from the environment becomes necessary so we can develop manageable analyzes for an informational and mobility plan for the proactive management of mobility throughout its social scope.

This kind of information can be captured through automated sensors and microcontrollers using IoT, which according to GARUMAN (2014), in essence IoT is an environment that gathers information from various devices such as computers, vehicles, smartphones, traffic lights, and almost anything with a sensor and internet connectivity, and from applications such as social media application like Twitter to an e-commerce platform, from a production system to a traffic control system.

In Brusque, Santa Catarina/Brazil, we have many traffic bottlenecks which affects directly on our urban mobility, we have a large variety of thermal readings in different areas of the city, which we don't have data collected and processed in reports to help the decision making to improve the life quality, may it be by planting trees or redirecting traffic, and so on. The main objective is to provide detailed reports of a micro and macro scenario of the city, to help and justify each change made for the best interest of the society. Collecting data do provide a historic database to better understand and generate precise forecasts for the future.

Our main goal with this research is to develop an open source meteorological monitoring centre device, CMMOS for short, which can gather environmental information such as, weather, humidity, traffic flow and $\mathrm{CO} 2$ concentration along with temperature and luminosity. The CMMOS device will capture all of this information and send it through the internet to a cloud hosted API server for later processing. The CMMOS development targets no financial end, as an open source project; at the end of it all of the schematics will be available online along with the instructions and coding that went into developing it. We aim to gather such information from the environment, compress it and send it to a web server application, API, at a periodicity of 5 minutes between readings, but also counting on triggering events such as when there is a great discrepancy of information detected in on each of the sensors readings, triggering an alarm data push. 


\section{BODY OF PAPER}

IoT contributes as a necessary mean that can generate useful data, history, trend and forecast to support and realize the changes that benefit the society as a whole and not only specific points, being that often we do not have a managerial base, according to (BRUTON, 1975), the first application of computers to the transportation planning process was the tabulation of data collected in fields, with IoT we aim to make the technology and application of computers to be able to collect this data more accurately and safely .

\subsection{Internet of Things}

Internet of Things or IoT is nothing more than gathering information between devices capturing information already present in the environment in which they are inserted to, transform that information that are being, in theory, wasted; in useful information for consumers or producers and suppliers.

According to (MANTELLATTO, 2013), basically, the concept of IoT applies to communication between objects, and between them and the internet, be their communication physical or virtual. For communication of these objects various features can be used, for example the wireless network or satellite mobile data plans.

We can say that IoT is an extension of the Internet that we know today, it offers the development of applications and services with great social benefits, something complex to implement in yesteryears due to the technological restrictions, but within the new concept of IoT, these restrictions are overcome, and a huge diversity of necessities will be attended and technologies will be developed to realize this type of network connection.

\subsubsection{Micro Controllers}

Within the branch of Internet of Things essential to use are those of micro controllers to automate routines and data capture that do not have a specific equipment to perform the desired data collection and the micro controller is nothing more than microchips pre-programmable which execute infinitely repeated instructions commands while the chip is being fed with energy, in the case of the CMMOS Open Source Weather Monitoring Center which we're developing, every 5 minutes the micro controller picks up information from the sensors connected to it and send it to an API for data collecting and processing.

According to BRAGA (2017), a microcontroller consists of an integrated circuit that gives us the idea that it is possible to assemble a single circuit or a component with a complete circuit that performs a certain function. For example, we have integrated circuits that perform functions of amplifiers, voltage regulators, logic functions, etc.

\subsubsection{Arduino}

The structure of the Arduino is composed of a design and a product where the electronic platform is open source; a single-board electronic hardware aiming for prototyping projects which makes use of an integrated micro controller with input / output support built into its structure. It has a standard programming language, mainly $\mathrm{C} / \mathrm{C}++$. The goal of the Arduino project is to create tools and new products that are affordable, affordable and easy to use by amateurs and technology enthusiasts, especially those who would not have access to advanced controllers and complex tools that require high investment in academic training and high purchasing power.

\subsection{Sensors}

Sensors are electronic, electrical or induction devices that convert information from the environment in which they are inserted into interpretable data capable of analysis, projection of trends and thus generating managerial information. The sensors used will be the following: temperature and humidity sensor DHT22; light sensor LDR 5mm; gas sensor model MQ-7 Carbon monoxide. With this range of sensors we will be able to chart the data and provide a basis for historical information to chart future trends. 


\subsection{Materials and Methods}

The applied research in the development of this work will be exploratory, whose main method is the field research, interviews with specialists and bibliographical analyzes by the researchers, making them thus proficient in the subject and able to develop his solutions in the desired field of studies.

The research population consists of the information elements that exist in urban society, be they meteorological or urban mobility wise and the data collection will be through the use of the micro controller developed during the course completion work, using sensors connected to an Arduino micro controller to collect the data present in the environment.

The data we gather through the micro controllers and the sensors will be dispatched via Wi-Fi internet connection to an API hosted at a Web Server with an REST construction awaiting the data packets, to later then break them into their respective categories in the database and further analyze and cross calculate the averages through history better understanding future tendencies.

Once the micro controller is fired up it will boot and start its programming cycles and to gather the sensors information, for the purpose of testing the first data were collected and dispatched in an interval of 5 minutes to the API to collect. The dataset was compiled in JSON, JavaScript Object Notation, which is an international standard for data object compiling of information and compression of data packets, the present structures is as follows:

\{

"CMMOSId":"9563533343035131F070",

"CMMOSType":"ARDUINO",

"DtHr":"24-11-2018 04:30",

"Tmp":29,

"Hmd":70,

"Glp":163,

"Lum":65

\}

The data structure begins with a curly bracket, followed by the identification name of the data being sent in between quotation marks, to separate the id from the value it is utilized a colon and then the data reading provided by the sensors in quotation marks as well. To separate an Id and value from one another is utilized a comma, and to close the package, the closure of the initial curly bracket.

In the data example above we can interpret it as it follows: "CMMOSId" is the identification from the micro controller, which is a unique hexadecimal code that identifies the sender to the server; "CMMOSType" is the type or brand of micro controller which is sending the data so we can differentiate the precision of readings depending on which CMMOS device will be uploading the data, in this case was an Arduino microcontroller; "DtHr" is an acronym for Date/Hour which is the identification for the timestamp at when the data was gathered in a 24 hour format and Day/Month/Year date format; "Tmp" is the temperature reading in Celsius degrees; "Hmd" is the humidity reading in percentages of water pressure relative to water steam pressure; "Glp" is the reading from the carbon monoxide sensor which reads the concentration of the particle in parts per million in a molecule of Oxygen and finally "Lum" is the luminosity reading in lumens, which is an international standard for reading light incidence.

The API, Application Programming Interface, developed to collect the data sent by the CMMOS is developed in GO Language, a programming language developed by Google, which was chosen for its low level of language branch and faster performance in data processing than its competitors, such as NodeJs. It was the main programming language we found viable to do the raw processing of numerous requisitions the CMMOS are capable of sending per controller cycle without losing any server performance and response time.

The construction method adopted was REST, Representational State Transfer, which means it utilizes HTTP, Hypertext Transfer Protocol, by the means of GET and POST methods to receive and insert the data respectively into the database.

To store the data we collect we chose the MariaDB database, which is a free, community developed, relational database that branched from the MySQL project and can handle the data flow we are expecting and can provide the performance we expect when we request the calculations and return of datasets to compose our graphs in our dashboards. The MariaDB works around with the Amazon Web Service related service, ARDS, 
Amazon Relational Database Service, the API connects to the database through an ORM, Object-Relational Mapping, via IP address to make all operations necessary.

To host our API and Database we chose to opt for the Amazon Web Services provider for their reliability and delivery speeds and for an all-around complete solution to host our server. On our AWS environment, it is running a Linux operational system virtual machine supporting the API and database which are going to receive and process all the data we manage to gather.

\section{CONCLUSION}

The development of the CMMOS assumes that the use of the information society produces for its own benefit must be accessible to all and in a transparent manner, it must be attractive and at a fair price to instigate the use of its information by the municipality and the municipality, the original idea came from the SMIGHT Smart Stations installed in the municipality of Brusque by the partnership of Brusque City Hall with the University Center of Brusque - UNIFEBE and their sisters in the district of Karlsruhe in Germany. Where each of these stations that has been installed in the city, there are currently three, high cost, with CMMOS and its open code design and physical structure, the cost may be more affordable and attractive, being easier to implement more solutions in different locations.

Thus, it is possible to have a municipal intelligence network capable of exponentially improving life in society, making use of information by creating a basis for a neural and analytical network structure capable of tracing and predicting trends based on the history and event of predecessor events, thus providing an assertive and well-founded prediction.

\section{ACKNOWLEDGEMENT}

With this research we have obtained indicators of more sustainable and accessible alternatives for more assertive environmental management, with a well-founded historical data line, in order to create a neural network capable of predicting historical-based trends. In sight of this technology and its capabilities, we aim to explore and extend the range of collectable information, such as flow indicators and traffic intensity; through sensors so that we can cross-reference and process this information to better understand our environment at the micro level.

\section{REFERENCES}

Braga, N. C. (2017), O básico sobre microcontroladores - Instituto NBC, 2017. Available: http://newtoncbraga.com.br/index.php/eletronica/52-artigos-diversos/13263-o-basico-sobre-os-microcontroladoresparte-1-mic139 [accessed 20 Nov 2018]

Breen, M., Geragh, A. and Punch, P. (2007). Cite it Right - Guide to Harvard Referencing Style - Second Edition, University, Glucksman Library. University of Limerick. Available at https://www2.ul.ie/pdf/467372218.pdf [01.07.2016].

Bruton, M. J. (1975), Introduction to transportation planning. London: Hutchinson \& Co., 1975. chapter 7, p. 154-155.

Garuman, G. (2014), IoT é um grande e confuso campo à espera de explodir - ComputerWorld from IDG. Available: https://computerworld.com.br/2014/11/25/iot-e-um-grande-e-confuso-campo-a-espera-de-explodir [accessed 20 Nov 2018].

Mantellatto, R. (2013), IoT Internet das Coisas - DEXTRA. Available: http://dextra.com.br/pt/blog/iot-internet-das-coisas/ [accessed 20 Nov 2018]. 